\title{
Automated Generation of a Digital Twin of a Process Plant by Using 3D Scan and Artificial Intelligence
}

\author{
Matthias GRAU, Wjatscheslaw KOROL, Johannes LÜTZENBERGER and Josip \\ STJEPANDIĆ ${ }^{1}$ \\ PROSTEP AG, Germany
}

\begin{abstract}
The simulation of production processes using a Digital Twin is a promising tool for predictive planning, analysis of existing systems or processparallel monitoring. In the process industry, the concept of Digital Twin provides significant support for process optimization. The generation of the Digital Twin of an already existing plant is a major challenge - in particular for small and mediumsized enterprises. In this sense, the twinning of the existing physical environment has got a particular importance due to high effort. Shape segmentation from unstructured (e.g. point cloud data) is a core step of the digital twinning process for industrial facilities. This is an inherent issue of Product Lifecycle Management how to acquire data of existing goods. The practice of Digital Twin is described based on object recognition by using methods of Machine Learning. The exploration of the pipeline semantics presents a particular challenge. The highly automated procedure for the generation of Digital Twin is described based on a use case of a biogas plant. Commercial deployment, pitfalls, drawbacks and potential for further developments are further explored.
\end{abstract}

Keywords. Digital Twin, Process Plant, Object Recognition, Indoor Object Acquisition, Simulation, Transdisciplinary Engineering

\section{Introduction}

The concept of Digital Twin consists of generation, maintenance and use of the virtual surrogate of an object or system during its entire lifecycle. The aim of this approach is to achieve a highly reliable product/system definition, verification, and validation including the modes of failure. By gathering and processing real-time data, the real system can be monitored with its Digital Twin, while (potential) problems can be detected, communicated with the real system, and dealt with [1]. The concept has been applied in the manufacturing domain originally, but is increasingly used in other domains like process industry and became a pillar of Product Lifecycle Management (PLM). It is a concept that requires a transdisciplinary approach for its creation, implementation, and use. Even when applied in the manufacturing domain predominantly, business knowledge as well as human behavior knowledge are needed, next to the technical knowledge. Without collaboration between people from these different disciplines, the Digital Twin concept cannot fully be exploited [2].

\footnotetext{
${ }^{1}$ Corresponding Author, Mail: josip.stjepandic@prostep.com.
} 
From the early beginning, Industry 4.0 has facilitated digital achievements as the pillars of its development [3]. Due to fact that all data are available in digital format, and sensors are inbuilt into the industrial spaces, the main pre-requisites for Digital Twin in the area of manufacturing are fulfilled. A mirroring or twinning of systems between what existed in real space to what existed in virtual space and vice versa becomes possible and meaningful. Using the emerging extensive simulation capabilities, it became feasible to perform realistic tests in a virtual environment. Furthermore, the overwhelming rise of artificial intelligence, primarily expressed by techniques such as Machine Learning, made a significant contribution to the further progress of the Digital Twin [4].

Following these technical advancements, manufacturing industry has broadly implemented Digital Twins in the past few years for specific tasks, for example, the monitoring and the predictive maintenance of long-lasting goods in service [5]. In this sense, the twinning of the existing physical environment has got a particular importance. This is an inherent issue of PLM how to acquire data of existing goods.

To acquire the necessary data of industrial or process plant, three approaches can be considered: (i) manual measurement and manual remastering, (ii) acquisition of geometry by a device (e.g., 3D scanner) followed by manual remastering, and (iii) acquisition of geometry by a device (e.g., 3D scanner, 360 degree camera) with a subsequent object recognition [6]. The latter provides the foundation of this paper.

The outline of the paper is as follows. In section 1, the background of the generation of a Digital Twin is introduced. In section 2, the process definition for object recognition in a process plant is explained. Results for a process plant are presented in section 3 . Discussion of the challenges that still exist in the process domain is presented in section 4. The paper ends with a brief conclusion and outlook.

\section{Background}

Process engineering facilities are constantly being rescheduled and rebuilt in order to increase their productivity or to produce other products with them. They need regular maintenance. In addition, when carrying out incident analyzes, the authorities check whether they can be operated safely and evacuated.

Facilities are often bought and sold again without the entire know-how being handed over to the new owner. In many cases, the know-how is stored as expert knowledge in the heads of the operating and maintenance staff. It is lost when the staff retire or are replaced by employees from outside the companies who are not very familiar with the operation of the facility.

Therefore, there are many good reasons for operators to precisely record the actual status of their facilities. This actual status never exactly corresponds to the planning status. Already during the construction of the facility deviations appear and / or many details are not planned at all [7]. Therefore, many operators try to bundle the information on their existing facilities in a Digital Twin.

The Digital Twin is a virtual representation of a real existing facility. It can contain different information and have different users, depending on whether it is to support development, planning, assembly, production or service. The operators are faced with the question of how they can set up this digital substitute with a reasonable expenditure of time and money.

Driven by a tremendous development in area related to computer vision, a plethora of object recognition approaches and methods were developed recently for different 
purposes. Although photogrammetry still plays an important role, a trend to point cloudbased approaches is obvious which is the basis for object recognition algorithms. For industrial application with rough environmental conditions (dirt, smoke, darkness, dust, vibration), it looks like the best compromise between costs, accuracy, acquisition speed and robustness. A scanner can be used as a terrestrial and mobile device (carried by human, car, drone) [6]. More recently, promising approaches for use of consumer 360 degree cameras were developed. In order to prepare the point cloud for a feature-based process of planning and simulation, the objects it contains must first be recognized and a CAD-based structure must be created.

Here, the challenge is how to embed the object recognition in the process of virtual 3D layout planning in a built environment as well as which findings and results can be expected. Realistic 3D layout models need to be created using point clouds acquired by commercial devices and prepared for object recognition with methods of Artificial Intelligence (AI) considering the strict data quality requirements. The generic procedure, the assessment of the available object recognition frameworks and the solution approaches are described in the previous reports [8][9]. Seamless, robust, (semi-) automatic workflow of primarily standard, modular components with low user assistance is of particular interest in order to achieve high efficiency. A proper quality assessment and error handling is necessary to facilitate automation of the entire process [10]. Once a highly reliable CAD model of a plant is created, there are several options to transform and export it to further tools in order to generate a Digital Twin.

Due to their functional importance, pipelines are crucial structures in process plants. They consist of branched structures organized in dense and complex cylinder configurations. Although pipes are merely cylindrical primitives which can be easily defined by their axis and radius, they are often combined with additional components such as flanges, valves, inlets, elbows, tees, etc. 3D scanning and recognition of pipelines is challenging due to small pipe surfaces and their intricate structure causing large selfocclusions, missing parts and insufficient sampling [11].

\section{Process definition}

Complex procedures as described in previous research [8][9] usually are being controlled by appropriate workflow software. Prior of such an implementation, it must be defined how the process steps scan, object recognition and generation of Digital Twin (Figure 1) interact with each other [12]. Basically, that are tasks for different user profiles because the present technology does not provide the performance to collect entire functionality for generation of Digital Twin in one device (e. g. portable computer). The tasks scan, object recognition, design engineering (to provide the requested change) and process planning (generation of Digital Twin) can be seen functionally independently of each other; with data exchange among singular steps [13].

The object acquisition must be provided at the plant site by a device (scanner or camera, terrestrial or mobile) [14] and the raw data are transferred by a secure web connection or disk to the next instance which provides data processing (object recognition, feature recognition, translation). After the full data model of the plant was created, it is sent to the design and engineering which complete the required change of the process system. Finally, the data set ca be sent to the plant engineering to conclude simulation, adaption and optimization (Figure 1) [15]. 
While some steps must be done manually (point cloud preparation, noise filtering, outlier removal, feature extraction, and almost the entire training), the workflow has to ensure an automated execution of all remaining steps. This is in particular true for the most time-consuming steps: execution of segmentation and execution of object recognition. The flexible, modular handling of the object recognition framework must also be provided [16].

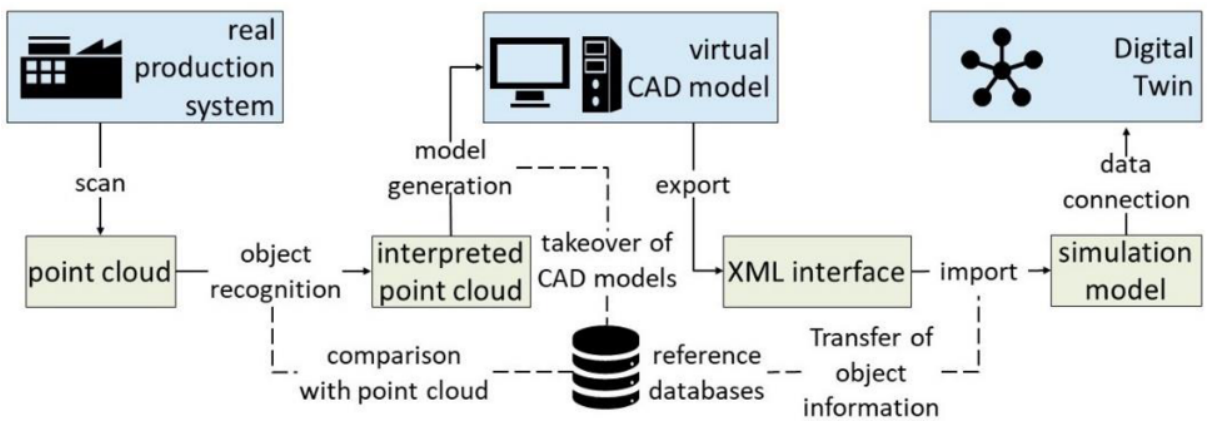

Figure 1. Workflow generation of Digital Twin [15].

The most manual work is expected on the segmentation of complex scenes which promises the highest rationalization potential, although no support from public directories can be expected [17]. A successful segmentation anticipated a reduction in data volume of point clouds with a factor of 1.000 and more between the rough point cloud and the segmented point cloud focused on segments for further processing can be achieved. During these manual steps, the model clean-up, the noise filtering and the outlier removal are conducted. The extraction of models and portions for training of recognition respectively segmentation occurs at this point of time. Finally, the point cloud must be exported in the desired format (e. g. e57) [18].

From this point on, the process runs automatically apart of training which is not mandatory for each data set [19]. Training is necessary in a sufficient extent to provide the test base for the later testing [20]. Mesh reconstruction and point cloud segmentation are implemented as separate utilities for the object recognition framework. To collect all automatic running steps, a user front-end was built. It controls the semantic segmentation, cluster extraction and cluster classification (recognition of singular objects). A viewer is included which allows the visualization of each intermediate result [21].

Segmentation is conducted manually or by supervised training which is preferred for complex scenes. The first issue is the preselection of smaller agglomerations in the entire point cloud which are assumed to contain an object. Such clusters can be covered by a bounding box like a packing box for a product in the right position and orientation. It helps in the assessment of results, too. The segmentation module is being trained to subdivide the entire scene in nine categories: roof, wall, floor, pipelines, structure, vessels, transport, auxiliary, and noise. For a process pant, pipelines, apparatus and connected devices are of a particular interest and can be considered separately [15].

Before the object recognition can start, the objects of interest must be available in the system library. Since they are usually not included in the standard database such as ModelNet, an additional reference library must be set up that contains the objects of interest. This is performed in two ways: either by using CAD models with a good data quality [22](e.g., vendor library) or exemplary point cloud models. To ensure the similar properties of both model types, CAD models are transformed in point clouds using a virtual scanner which is stochastically moved around the object. 
Singular point clouds models must be further transformed in order to prepare an adequate input for object recognition procedures, depending on the object recognition framework used [9]. In case of the voxel-oriented framework, the point cloud must be translated into a corresponding voxel model. The side length of a voxel plays a significant role here. Tests have shown that a variable length which is related to the object shape provides the best results. Although the start model already has been simplified, a further simplification occurs during the translation to a voxel model which hardly can be prevented. If the simplification dilutes a local feature (e. g. an auxiliary device), then sufficient recognition results cannot be expected. Rather, it is to be feared that a wrong object will be recognized. Therefore, when building the sample models, care must be taken to ensure that the distinctive features are considered. In case of a pipe, the centerline and the radius are such distinctive features.

An exemplary procedure in three steps (segmentation, clustering, recognition of object in singular clusters) has been trained with up to 1000 epochs, the developed framework can be tested using real-life data extracted from the process plant.

\section{Results}

After the new integral DigiTwin tool was previously used for the generation of the Digital Twin in manufacturing [8], it was recently tested for a biogas plant located in north of Germany (Figure 2). In first case, an optimization of material flow was performed with the output of object recognition. The data of an existing biogas plant were necessary for training, operation, maintenance, and reconstruction.

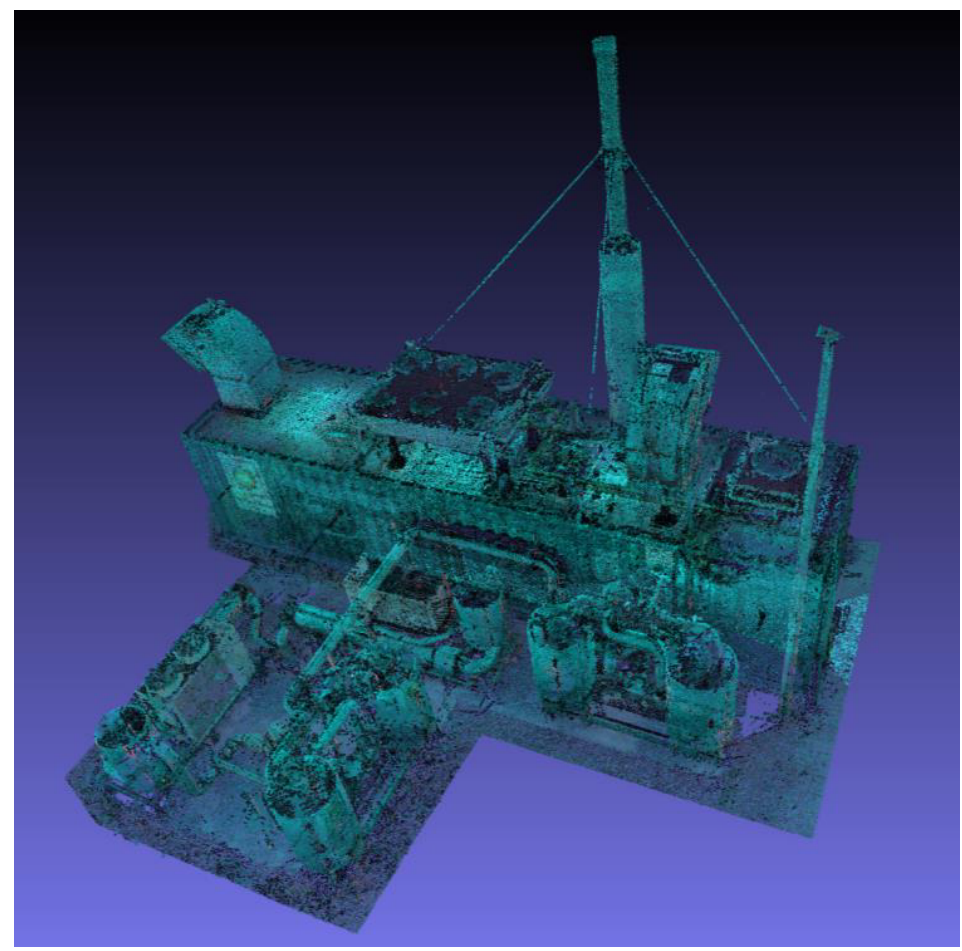

Figure 2. Biogas plant scanned with different devices. 
With regard to virtual training, maintenance or operation of a system, the topic of virtual reality becomes decisive. It must be concurrently fed by data. This is usually not a problem for new systems, as a later virtual operation can be included in the planning of the system. The as-designed models can be linked with piping diagrams and then enriched with sensor data from the system. For already existing systems, 3D models, if available, are often obsolete due to maintenance work, repairs or modernization activities and can no longer be used for the use of virtual applications. The effort to create a suitable $3 \mathrm{D}$ model is associated with great effort and correspondingly cost-intensive.

In all industrial cases, the object recognition of objects available in the library was of the particular importance. The models were manually prepared and put in the tool chain as described in the previous section. The overall recognition rate is high, although the environmental impact often was negative. As a rule, more than 75 percent of all objects can be truly recognized. However, the recognition procedure is sensitive and needs to be stabilized. Provision of bounding boxes for singular objects of interest is helpful for repair of missing and wrong oriented objects.

In order to verify this approach for the recognition of pipelines, experiments with the point cloud from the plant construction (biogas plant) have been conducted. This method is only based on the registered industrial point cloud itself regardless of the varying point densities [13]. Such industrial point clouds may contain defects such as occlusions and sparseness. In total, the results were acceptable, but disparate in detail. Good results were achieved in the segmentation phase automatically and robustly. Pipeline easily can be separated from the remaining components of a plant. In highly dense industrial spaces, class segmentation of cylinders, I-beams and valves that have easily distinguishable geometric patterns in the processing unit, warehouse and cranes work well.

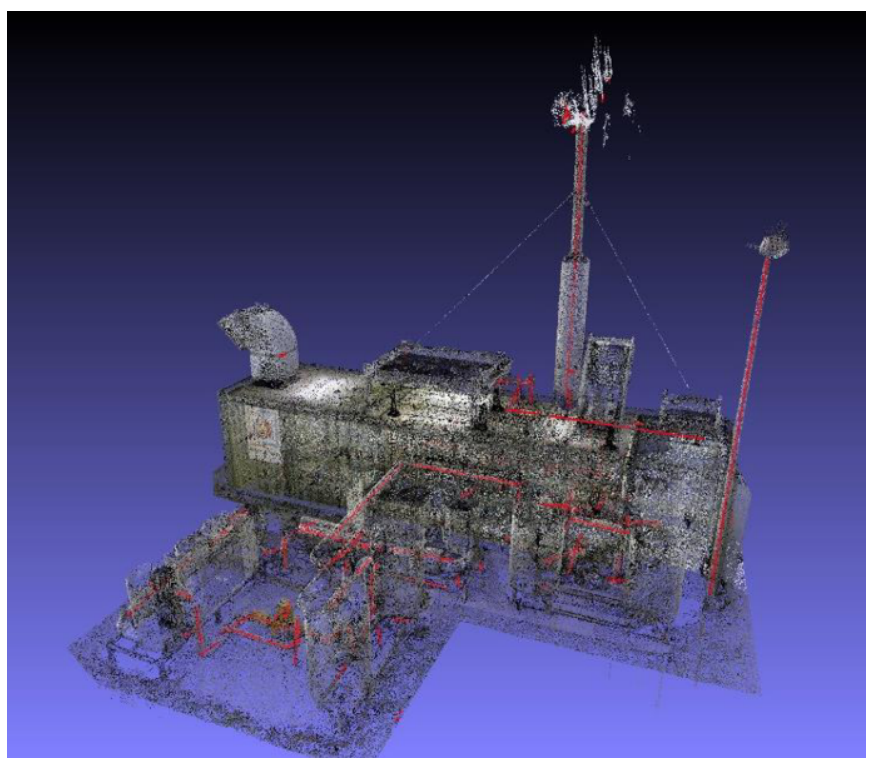

Figure 3. Results of the object recognition in a biogas plant.

The focus of a biogas plant lies in the recognition of the pipelines including their semantics (Figure 3). For this task, a specific extension of the object recognition framework was developed. The process step segmentation runs as previously described. 
The step clustering consists of the identification of the singular pipe segments which are subsequently added to pipelines. By detection of the corresponding pipe radius, the centerline of the pipeline can be derived. If all segments and control elements (flanges, valves, inlets, elbows, tees etc.) can be found, the pipeline can be completed. The integration of PI\&D diagram which is a pre-requisite for a operating license is not performed in this stage yet.

Figure 2 shows the point cloud model of the entire biogas plant which was obtained by scans from different devices with a total volume of 57 GB for the entire scan project. Such package is difficult to handle and cannot be used as an active environment in the conventional CAD systems. Portions of such point cloud can be imported as passive environment.

Figure 3 shows the extracted pipeline with its recognized centerlines and pipe radius respectively which would be subject of redesign and optimization. Worth to note, that the entire data volume was reduced from 57 GB for the entire plant to only $22 \mathrm{MB}$ for the pipeline. These intermediate results provide a good basis for design work on reconstruction and optimization since leading CAD systems can easily handle a smaller amount of point cloud models. This outcome is obtained in a neutral format and can be exported to each proprietary CAD format for further design work. Most issues occur on elbows which are often recognized in a false orientation. Therefore, the additional object recognition algorithms were necessary.

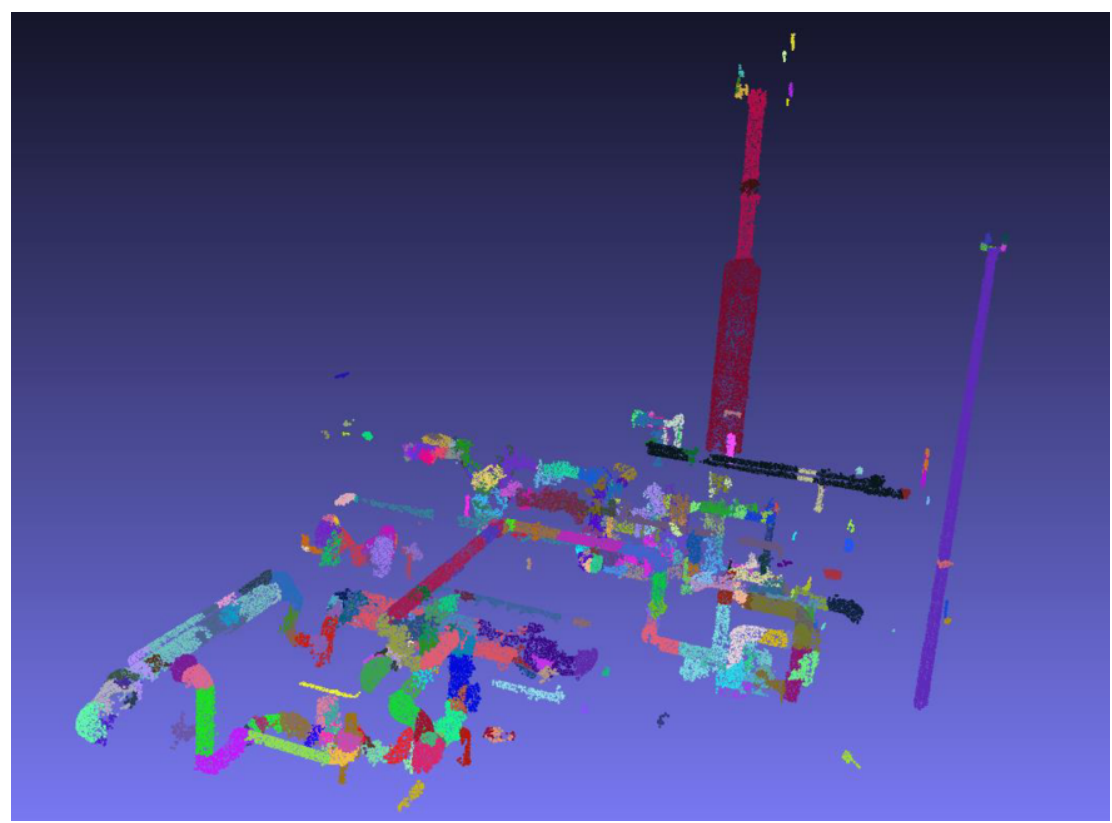

Figure 4. Recognition of singular pipeline segments.

Figure 4 sets the focus on sigular pipe segments which are marked in distinctive colors. However, a principal distinction between a pipe and an apparatus which can have an identical cylindrical shape must be done in a specific case. The recognition of all segments, devices and apparatus is the pre-requisite for the recognition of the pipeline semantics. 


\section{Discussion}

The successful generation of Digital Twin paves the way for a full digitalization of the process plants. Although there is still no legal requirement in many countries, a 3D model of the plant is an important aid in development, production and operation of a plant. The approach presented here is capable to deal with complex real-world industrial facilities, such as highly dense biogas plant. Assuming that a searched object is known, either in the publicly available or in the private library, there is a high probability that it will be recognized in the correct position and orientation. The distance deviation of the absolute position in space is acceptable for this purpose since it remains in the tolerance range known from literature [13]. Manual rework in a small extent can be always expected for each data set, indeed.

Based on these tests, several improvements must be taken into account for the next development steps:

- The huge amount of input data could be significantly reduced by a better scanning procedure, if constructional domain knowledge can be used for this purpose. That could be achieved by a pre-scan of objects of interest as known from autonomous driving. The camera-based approach is especially promising [6].

- By using low-cost devices (e. g. augmented reality glasses, 360-degree camera), the usability and the acquisition speed could be improved and the costs reduced. This would prevent the impact of occlusion. However, the concern of the low position accuracy still remains [6].

- The final results could be improved by extension of the object library, for example by a blend with further libraries (CAD standard part libraries). Industry circles and associations could provide their data.

- The recognition of pipelines in fully variety needs to be considered. The pipe classification is noisy and should be reinforced by robust clustering and graph-based aggregation techniques to compute a coherent pipe model [23].

- While the training requires so much effort, its automation would drastically reduce the processing time and increase the overall efficiency.

- Integration of P\&ID drawings and non-geometrical information would improve the confidence and the acceptance by the regulatory bodies.

- While the object recognition basically is a probability calculation, it needs an extensive and quantitative comparative performance evaluation in order to meet the requirements from the industry.

The commercial use of the described procedure and tool for generation of Digital Twin is provided by the portal OpenDESC.com which is in service for 23 years. It provides service for transfer and translation of the auxiliary data (primarily CAD) in the industrial supply chains. It offers services globally and is used by more of 100 customers located worldwide [24].

A deployment schema for generation of Digital Twin is depicted in Figure 5 as a collaborative procedure where the tasks scan, object recognition and process planning/layout design are undertaken by different teams. The scan is conducted on the customer's premise, either by customer or a service provider. Then point cloud data are sent to OpenDESC.com where object recognition is done as described in section 2. Results can be transmitted to the plant engineering / process planning where the Digital Twin will be finally completed. 


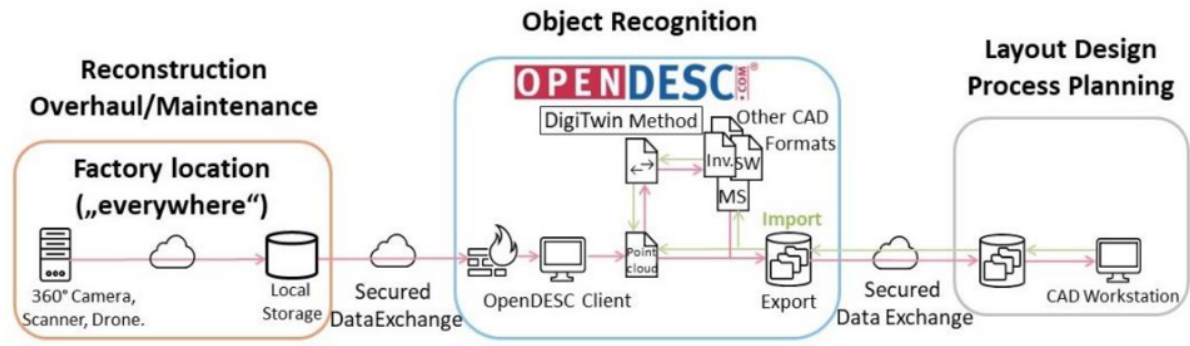

Figure 5. Workflow for generation of Digital Twin as a service.

\section{Conclusions and outlook}

The affordability of object reconstruction with semantic enrichment needs to be primarily justified in terms of cost-benefit [25] in comparison with the manual acquisition. If the object recognition as shown here in an use case from the biochemical industry is successful, the justification is easy. However, the drawbacks must be considered to prevent a use case with no recognition (e. g., on significant occlusion which cannot be compensated). Theoretically, in highly dense industrial spaces data acquisition could be made impossible both by a missing accessibility though the acquisition device and the poor recognition caused by incomplete point cloud and occlusion [26]. Therefore, such obstacles can only be resolved by an incremental scanning (e. g. by a highly flexible mobile device which can access each segment of a plant) [27].

Because the Digital Twin is subject to frequent changes, the question arises of how its consecutive updates (e. g. replacement for an apparatus in a larger hall) can be conducted without restraint to repeat the entire process described above [28]. A simple procedure need to be developed in which the user company is enabled to undertake the initial part of the update process itself. For this purpose, an application is being developed with which the partial spaces of the hall to be updated in the Digital Twin can be scanned using the camera of a standard smartphone. The application will guide the user during the recording process, so that handling can be carried out quickly and easily. The scan recorded with the application is further processed automatically to a high degree and the Digital Twin is updated. With the extension of the object recognition for the part of a model to be developed, the existing Digital Twin is analysed and changes are automatically overlayed.

\section{References}

[1] C.K. Lo, C.H. Chen, R.Y. Zhong, A review of digital twin in product design and development, Advanced Engineering Informatics, Vol. 48, 2021, 101297.

[2] N. Wognum, C. Bil, F. Elgh, M. Peruzzini, J. Stjepandić, W. Verhagen, Transdisciplinary engineering research challenges, Advances in Transdisciplinary Engineering, Vol. 7, 2018, pp. 753-762.

[3] C. Zhang, Y. Chen, A Review of Research Relevant to the Emerging Industry Trends: Industry 4.0, IoT, Blockchain, and Business Analytics, Journal of Industrial Integration and Management, 2020, Vol. 5, No. 1, pp. 165-180.

[4] R. Wallis, J. Stjepandić, S. Rulhoff, F. Stromberger, J. Deuse, Intelligent utilization of digital manufacturing data in modern product emergence processes, Advances in Transdisciplinary Engineering, Vol. 1, 2014, pp. 261-270. 
[5] Y. Wang, S. Wang, B. Yang, L. Zhu, F. Liu, Big data driven Hierarchical Digital Twin Predictive Remanufacturing paradigm: Architecture, control mechanism, application scenario and benefits, Journal of Cleaner Production, 2020, Vol. 248, 119299.

[6] M. Sommer, K. Seiffert, Scan Methods and Tools for Reconstruction of Built Environments as Basis for Digital Twins, in J. Stjepandić et al. (eds.) DigiTwin: An Approach for Production Process Optimization in a Built Environment, Springer Nature, 2021, in press.

[7] Z. Pučko, N. Šuman, D. Rebolj, Automated continuous construction progress monitoring using multiple workplace real time 3D scans, Advanced Engineering Informatics, Vol. 38, 2018, pp. 28-40.

[8] M. Sommer, J. Stjepandić, S. Stobrawa and M. von Soden, Automatic Generation of Digital Twin Based on Scanning and Object Recognition, Advances in Transdisciplinary Engineering, Vol. 10, 2019, pp. 645-654.

[9] B. Salem, J. Stjepandić and S. Stobrawa, Assessment of Methods for Industrial Indoor Object Recognition, Advances in Transdisciplinary Engineering, Vol. 10, 2019, pp. 390-399.

[10] L. Wagner, Kein Digital Twin ohne digitale Durchgängigkeit. Zeitschrift für den wirtschaftlichen Fabrikbetrieb, 2020, Band 115, pp. 43-45, DOI: 10.3139/104.112315.

[11] L. Cheng, Z. Wei, M. Sun, S. Xin, A. Sharf, Y. Li, B. Chen, C. Tu, DeepPipes: Learning 3D pipelines reconstruction from point clouds, Graphical Models, 2020, Vol. 111, 101079.

[12] O. Kuhn, H. Liese and J. Stjepandic, Methodology for knowledge-based engineering template update, IFIP Advances in Information and Communication Technology, Vol. 355, Springer, Berlin Heidelberg, 2011, pp. 178-191.

[13] Z. Ma, S. Liu, A review of 3D reconstruction techniques in civil engineering and their applications, Advanced Engineering Informatics, Vol. 37, 2018, pp. 163-174.

[14] D. Moon, S. Chung, S. Kwon, J. Seo, J. Shin, Comparison and utilization of point cloud generated from photogrammetry and laser scanning: 3D world model for smart heavy equipment planning, Automation in Construction, Vol. 98, 2019, pp. 322-331.

[15] J. Stjepandić, M. Sommer, Object Recognition Methods in a Built Environment, in J. Stjepandić et al. (eds.) DigiTwin: An Approach for Production Process Optimization in a Built Environment, Springer Nature, 2021, in press.

[16] E. Ostrosi, J. Stjepandić, S. Fukuda and M. Kurth, Modularity: New trends for product platform strategy support in concurrent engineering, Adv. in Transdisciplinary Engineering, 2014, Vol. 1, pp. 414-423.

[17] E. Agapaki, I. Brilakis, CLOI-NET: Class segmentation of industrial facilities' point cloud datasets, Advanced Engineering Informatics, 2020, Vol. 45, 101121.

[18] J. Stjepandić, S. Bondar, W. Korol, Object Recognition Findings in a Built Environment, in J. Stjepandić et al. (eds.) DigiTwin: An Approach for Production Process Optimization in a Built Environment, Springer Nature, 2021, in press.

[19] K. Kawashima, S. Kanai and H. Date, As-built modeling of piping system from terrestrial laser-scanned point clouds using normal-based region growing, Journal of Computational Design and Engineering, Vol. 1, 2013, pp. 13-26.

[20] T. Czerniawski, F. Leite, Automated digital modeling of existing buildings: A review of visual object recognition methods, Automation in Construction, 2020, Vol. 113, 103131.

[21] C. Emmer, A. Fröhlich and J. Stjepandić, Advanced engineering visualization with standardized 3D formats, IFIP Advances in Information and Communication Technology, Vol. 409, Springer, Berlin Heidelberg, 2013, pp. 584-595.

[22] S. Bondar, C. Ruppert, J. Stjepandić, Ensuring data quality beyond change management in virtual enterprise, International Journal of Agile Systems and Management, 2014, Vol. 7, Nos. 3/4, pp. 304-323.

[23] R. Maalek, D.D. Lichti, R. Walker, A. Bhavnani, J.Y. Ruwanpura, Extraction of pipes and flanges from point clouds for automated verification of pre-fabricated modules in oil and gas refinery projects, Automation in Construction, 2019, Vol. 103, pp. 150-167

[24] S. Bondar, L. Potjewijd, J. Stjepandić, Globalized OEM and tier-1 processes at SKF, in: J. Stjepandić et al. (eds.) Advanced Concurrent Engineering, Springer-Verlag, London, 2012, pp. 789-800.

[25] C.T. Li, J.C.P. Cheng, K. Chen, Top 10 technologies for indoor positioning on construction sites, Automation in Construction, 2020, Vol. 118, 103309.

[26] A. Garcia-Garcia, J. Garcia-Rodriguez, S. Orts-Escolano, S. Oprea, F. Gomez-Donoso, M. Cazorla, A study of the effect of noise and occlusion on the accuracy of convolutional neural networks applied to 3D object recognition, Computer Vision and Image Understanding, 164, 2017, pp. 124-134.

[27] S. Bondar, B. Salem, J. Stjepandić, Indoor Object Reconstruction based on Acquisition by Low-cost Devices, Advances in Transdisciplinary Engineering, 2018, Vol. 7, pp. 113-122.

[28] Stjepandić, J., Sommer, M., Stobrawa, S.: Digital Twin: Conclusion and Future Perspectives, in J. Stjepandić et al. (eds.) DigiTwin: An Approach for Production Process Optimization in a Built Environment, Springer Nature, 2021, in press. 